\title{
Effect of Carbon fiber on the Physico-Chemical Properties of Conductive Butyl-Rubber Composite
}

\author{
F.S. Deghaidy \\ Department of Chemistry, Faculty of Science, \\ Suez Canal University, Ismailia - Egypt
}

This study focuses on the effect of the carbon fiber $(C F)$ concentration on the physico-chemical properties of Isobutylene and Isoprene Butyle Rubber (IIR) composites. The kinetic parameters for the vulcanization process of IIR/CF composites were calculated and discussed. The swelling mechanisms were identified; whereas, relative humidity and resistance during swelling as a function of carbon fiber content were measured. The effects of carbon fiber contents on some electrical and mechanical properties were investigated. SEM was used to support the observed trends. It was demonstrated that carbon fiber accelerates the vulcanization process by acting as a catalyst and improves the physico-chemical properties of IIR composites for practical applications. 


\section{Introduction}

Recently there has been a growing interest in polymer composites, where different fillers were used to improve the characteristics of the composite [1-13]. In spite of the fact that polymer exhibits good insulation properties, its electrical conductivity is important in many applications. In general, the electrical conduction process in carbon black polymer composites is complicated and depends on large number of parameters, mainly on filler concentration. As Carbon fibers (CF) filler has its own attractive properties, this study focuses on the effect of the carbon fiber (CF) concentration on the physico-chemical properties of Isobutylene and Isoprene Butyle Rubber (IIR) composites. In addition to the amount of loading $\mathrm{CF}$, particle size and structure, filler matrix interactions, swelling and processing techniques are key factors in determining the physical properties [2,4]. Analysis of the mechanisms of swelling in polymeric systems has received considerable attention in recent years because of the importance of the applications of polymers in biomedical, environmental and agricultural engineering and others $[3,14]$.

\section{Experimental Procedure}

All rubber mixtures were prepared on a two-roll mill of $170 \mathrm{~mm}$ diameter, working distance $300 \mathrm{~mm}$, speed of slow $18 \mathrm{rpm}$ and gear ratio 1.33. The various rubbers used in this work were prepared according to the recipes given in Table (1), where Polyacrylonitrile based CF was used. The average diameter of a single filament of the CF was $6 \mu \mathrm{m}$ while the CF density was $1800 \mathrm{~kg} / \mathrm{cm}^{3}$. Phosphoric acid $\left(\mathrm{H}_{3} \mathrm{PO}_{4}\right)$ was used for inhibition and/or retardation of $\mathrm{CF}$ oxidation during vulcanization process. An overall mixing time of 1 hour at $20{ }^{\circ} \mathrm{C}$ before adding $\mathrm{CF}$ was allowed to ensure homogeneity of conductive particles. Vulcanization was carried out between stainless steel platens at $175^{\circ} \mathrm{C}$ and pressure $300 \mathrm{kN} / \mathrm{m}^{2}$ for dwelling time $30 \mathrm{~min}$. Thin brass electrode was embedded into samples during vulcanization process. A circuit used for electrical conductivity measurements during vulcanization process was described elsewhere [4,13]. Mechanical properties of the samples were measured by the Shimadzu AGS-IKNA Tensilon. The morphology was studied by scanning electron microscopy (SEM),

The crosslinking density (CD) was calculated according to the equation, [12]:

$$
\mathrm{CD}=\frac{\left(m_{t}-m_{o}\right)}{m_{r} m_{o}}
$$

where $\mathrm{m}_{\mathrm{t}}$ is the mass of the swollen polymer at time $\mathrm{t}, \mathrm{m}_{\mathrm{o}}$ is the mass of dry polymer at time 0 , and $\mathrm{m}_{\mathrm{r}}$ is the mass of rubber used in phr.

The volume fraction of rubber $\left(\mathrm{V}_{\mathrm{r}}\right)$ was calculated according to the equation [13]: 


$$
\mathrm{V}_{\mathrm{r}}=\frac{\left(m_{t}-\lambda m_{o}\right)}{\rho_{r}} /\left(\frac{m_{t}-\lambda m_{o}}{\rho_{r}}+\frac{\beta_{o}}{\rho_{s}}\right)
$$

where $\lambda$ is the mass fraction of the insoluble component in rubber sample, $\rho_{r}$ and $\rho_{\mathrm{s}}$ are the densities of IIR and kerosene respectively and $\beta_{o}$ is the mass of the observed solvent (kerosene) corrected for swelling increment.

Table (1): Formulation of the recipes.

\begin{tabular}{|c|c|c|c|c|c|}
\hline Ingrediants (phr) & F0 & F3 & F6 & F9 & F1 \\
\hline Butyl rubber (IIR) & 100 & 100 & 100 & 100 & 100 \\
\hline Stearic acid & 2 & 2 & 2 & 2 & 2 \\
\hline Zinc oxide & 7.5 & 7.5 & 7.5 & 7.5 & 7.5 \\
\hline Processing oil $^{\text {HAF) black }}{ }^{\mathrm{a}}$ & 10 & 10 & 10 & 10 & 10 \\
\hline TMTD $^{\mathrm{b}}$ & 50 & 50 & 50 & 50 & 50 \\
\hline Carbon fiber (CF) $^{\text {fipO }}$ & 0 & 1 & 1 & 1 & 1 \\
\hline $\mathrm{H}_{4}$ & 1 & 1 & 1 & 1 & 1 \\
\hline Sulfur & 1.75 & 1.75 & 1.75 & 1.75 & 1.75 \\
\hline
\end{tabular}

(a)High abrasion furnace black, (b)Tetramethyl thiuran disulfide.

\section{Results and Discussion}

\section{Effect of Carbon Fiber on Vulcanization Kinetics of IIR composites}

The vulcanization time versus electrical conductivity of IIR composites with different concentrations of $\mathrm{CF}$ at $\mathrm{P}=300 \mathrm{kN} / \mathrm{m}^{2}$ and $\mathrm{T}=175$ ${ }^{0} \mathrm{C}$ are shown in Fig. (1), (at this temperature solid sulfur transforms to liquid sulfur consisting of $\mathrm{S}_{8}$ ring molecules and chain molecules with an average molecular weight of more than $10^{5}$ ) [15]. It is clear that the vulcanization begins during heating and depends on CF concentration. The vulcanization process shows an S-shaped profile shifted to shorter time scales with increasing $\mathrm{CF}$ contents. The behavior of vulcanization process can be divided into two regions: in the first one, the electrical conductivity increases rapidly. This may be attributed to ordering of the conductive phases and thermal activation causing rapid crosslinking density [13]. In the second stage, the conductivity slightly increases and then remains steady with time for about five hours. This plateau corresponds to completion of the vulcanization process [5].

It was found that the separation distance between conductive aggregates, (calculated according the equations given by Ref. [6]), decreases with increasing $\mathrm{CF}$ content \{Table (2)\}.This reflects the fact that the CF enhances the crosslinking density and increases the interfacial bonding of the fiber and matrix. 
The characteristic time constant $(\tau)$ as a function of CF contents can be estimated by the empirical formula:

$$
\left(\begin{array}{ll}
\sigma & -\sigma_{o}
\end{array}\right)=\left(\sigma_{m}-\sigma_{o}\right)\left(1-\mathrm{e}^{-\mathrm{t} / \tau}\right)
$$

where $\sigma_{m}$ and $\sigma_{\mathrm{o}}$ are the maximum and initial conductivity of the tested samples respectively and $\tau$ is the characteristic time constant depending on CF concentration.

The values of characteristic time constant as a function of $\mathrm{CF}$ content are listed in Table (2). In Table (2), the characteristic time decreases with increasing CF; this implies that the $\mathrm{CF}$ accelerates the driving force during vulcanization process and increases the reaction kinetics rate [5].

The mass transport of vulcanization can be estimated according to the empirical formula:

$$
\frac{\partial \sigma}{\partial t}=A C^{n}
$$

where, $\partial \sigma / \partial t$ is the rate of conductivity during vulcanization, $\mathrm{A}$ is the overall rate constant of conductivity and is an incorporating characteristics of the aggregation distribution and $\mathrm{CF}$ content, $\mathrm{C}$ is the concentration of $\mathrm{CF}$ (phr), and $\mathrm{n}$ is the diffusion exponent, which is indicative of the transport mechanism during vulcanization process. The value of $\mathrm{n}$ for both stages of IIR composites with different concentrations of CF are listed in Table (2). The values of $n$ in Table (2) indicate that, two main vulcanizing stages are defined. The first one with $\mathrm{n} \geq 1$ indicates the mechanism of ordering of the conductive particles. The second stage with $\mathrm{n} \geq 0.5$ indicates that the grain boundary of aggregates diffusion leads to optimum vulcanization process [6].

The vulcanization parameter $\left(\Delta \sigma / \sigma_{o}\right)$ is time dependent because of the systematic decrease of the porosity of the compact sample and increase of the crosslinking with the vulcanizing time. The activation energy of vulcanization process $(\mathrm{Q})$ as a function of $\mathrm{CF}$ content can be estimated by the following equation, [7]:

$$
\frac{\Delta \sigma}{\sigma_{o}}=A \exp \left(Q n / K T_{t}\right)
$$

where $\mathrm{Q}$ is the activation energy for mass transport during vulcanization, $\mathrm{K}$ is the Boltzmann constant and $\mathrm{T}$ is the Kilven Temperature. The values of $\mathrm{Q}$ of 
IIR composites during vulcanization as a function of CF content are recorded in Table (2). It is considered that the activation energy decreases with increasing $\mathrm{CF}$ content which may indicate the slower rate of kinetic reaction during vulcanization process of samples having lower content of CF [8].

Table (2): Separation distance before $(\omega)$ and after $\omega$ (t) swelling, characteristic time constant during vulcanization $(\tau)$, diffusion exponent (n) and activation energy during swelling $(Q)$ as a function of $C F$ content of IIR composites.

\begin{tabular}{|c|c|c|c|c|c|c|}
\hline $\begin{array}{c}\text { Sample } \\
\text { batch }\end{array}$ & $\begin{array}{c}\omega \\
\left({ }^{\circ} A\right)\end{array}$ & $\begin{array}{c}\omega(\mathrm{t}) \\
\left({ }^{o} A\right)\end{array}$ & $\begin{array}{c}\tau \\
(\min )\end{array}$ & $\begin{array}{c}\text { First } \\
\text { Stage } \\
(\mathrm{n})\end{array}$ & $\begin{array}{c}\text { Secon } \\
\mathrm{d} \\
\text { stage } \\
(\mathrm{n})\end{array}$ & $(\mathrm{Q})$ \\
$(\mathrm{eV})$ & \\
\hline F0 & 800 & 989 & 40 & 1.511 & 0.751 & 4.03 \\
\hline F3 & 603 & 636 & 29 & 1.233 & 0.702 & 3.16 \\
\hline F6 & 501 & 507 & 22 & 1.294 & 0.625 & 2.33 \\
\hline F9 & 313 & 315 & 14 & 1.326 & 0.543 & 1.47 \\
\hline F12 & 136 & 139 & 10 & 1.371 & 0.504 & 1.02 \\
\hline
\end{tabular}

Fig (2) shows the relationship between crosslinking density (CD) and volume fraction of rubber $(\mathrm{Vr})$ against $\mathrm{CF}$ content. It is clear that, the $\mathrm{CD}$ and $\mathrm{Vr}$ increase with $\mathrm{CF}$ content. The $\mathrm{CD}$ and $\mathrm{Vr}$ clearly pronounce that the $\mathrm{CF}$ improves the contiguity links between conductive aggregate [16].

\section{Electrical Resistance During Swelling and Mass Swelling Of IIR /CF Composites}

Fig (3) shows the effect of $\mathrm{CF}$ on the electric resistance of IIR composites during swelling in kerosene at $20{ }^{\circ} \mathrm{C}$. It is noted that at low content of $\mathrm{CF}$ the resistance increases linearly while at higher content the resistance slightly increases quasi-constant. It is clear that the swollen behavior of IIR composites strongly depends on the aggregation structure and the molecular mobility of the solvent, in other words on the degree of ordering. Thereby, the increase of resistance at low CF content is ascribed to the kerosene molecules, which form a layer structure and/or bimolecular lamellae with rubber matrix [9] especially samples F0 and F6. SEM photograph in Fig. (4a,b) shows that there is a black hole inside a rubber matrix due to the reaction of kerosene molecules with molecules of rubber composites for sample (F0), while sample (F6) is not affected by solvent molecules.

The mass swelling $[\% \mathrm{~S}(\mathrm{~m})]$ of the IIR /CF composites can be estimated according to equation: 


$$
\% \mathrm{~S}(\mathrm{~m})=\frac{m_{t}-m_{o}}{m_{o}} \times 100
$$

Fig (5) shows that mass swelling is increasing with time, but after certain period becomes constant.

The Mass equilibrium Swelling (MES) can be calculated by the empirical formula:

$$
\left(S-S_{o}\right)=\left(S_{m}-S_{o}\right)\left(1-\mathrm{e}^{-\mathrm{N} / \tau}\right)
$$

where $S_{m}$ and $S_{0}$ are the maximum and initial swelling of IIR composites respectively, and $\mathrm{N}$ is the characteristic swelling time constant which corresponds to MES at $\mathrm{N}=\mathrm{t}$. Therefore, the molecules of rubber will expand due to network structure change. While at high content of CF the molecules of kerosene cannot penetrate into chains of rubber matrix. Table (3) represents the change of MES (\%) with CF content. A decrease in the MES values with increasing the $\mathrm{CF}$ content is observed. This may be attributed to penetration of the kerosene molecules into the chains of the rubber matrix at low CF content (i.e. samples F0 and F3). Therefore, the molecules of rubber will expand due to net work structural change. While at high content of CF the molecules of kerosene can not penetrate into the chains of the rubber matrix. Thereby, it is concluded that $\mathrm{CF}$ improves the microstructure core and ordering the rubber matrix.

Table (3): Mass Equilibrium Swelling (MES) values of IIR/CF composites

\begin{tabular}{|l|c|c|c|c|c|}
\hline Sample batch & F0 & F3 & F6 & F9 & F12 \\
\hline MES \% & 930 & 860 & 720 & 540 & 400 \\
\hline
\end{tabular}

\section{Effect of Relative Humidity on Resistance of IIR composites as a function of $C F$ content}

Fig. (6) shows the relationship between relative humidity of IIR composites and electric resistance as a function of $\mathrm{CF}$ content. It was found that for low content of CF, especially samples F0 and F3, the resistance increases linearly with increasing humidity, while at higher CF (i.e. samples F6, F9 and F12), the electric resistance slightly increases with humidity. This is because at low CF content the resistance increases due to the formation of liquid film with conductive aggregates which acts like membrane. It is concluded that the IIR CF composites can be used as humidity sensor and shielding. 
Effect of CF on Tensile strength - Modulus, Elongation and Hardness of IIR composites

Fig. (7 a, b) tensile strength and tensile modulus of IIR composites as a function of $\mathrm{CF}$ content. It is clear that tensile strength and modulus are increasing with $\mathrm{CF}$ content. Fig. ( $8 \mathrm{a}, \mathrm{b})$ shows optimum elongation and hardness of IIR composites at different CF content. It is clear that the elongation and hardness increase with CF content. These results suggest that, $\mathrm{CF}$ improves the homogeneity by connective linkage among aggregates and refines the microstructure stability of IIR composite [15].

\section{Conclusions}

- $\mathrm{CF}$ acts as a catalyst, reduces the curing time, accelerates the driving force, reaction kinetic rate during vulcanization process and improves the microstructral network of IIR composites.

- $\mathrm{CF}$ inhibits the swelling and the mechanism of diffusion which is of non-Fickian type.

- IIR composites at low CF content can used as humidity sensor while at high CF content can be used as humidity shielding.

- The electrical properties are strongly affected by CF content.

- CF improves the tensile strength - modulus, elongation and hardness of IIR composites.

\section{Acknowledgment}

The author is very grateful to Dr. F.El-Tantawy, Suez Canal University, Faculty of Science, Department. of Physics, for his help during the experimental measurements and useful discussions.

\section{References}

1. G. Wu, Polymer Journal, 29(9), 705, (1997).

2. D. Cho, Polymer Journal, 29(12), 959, (1997).

3. T. G. Gopakumar, Polymer Journal, 29(11), 884, (1997).

4. W. Hopark, Polymer Journal, 28(5), 672, (1996).

5. D. Saraydin, Polymer Journal, 29(8), 631, (1997).

6. J. Shan, Polymer Journal, 29(7), 580, (1997).

7. M. okazaki, Polymer Journal, 31(8), 672, (1999).

8. H. Tagachi, Physica B, 270, 325, (1999).

9. E. Iguchi, Physica B, 270, 332, (1999).

10. L. Karasek, Polym. J., 28(2), 121, (1996)

11. D. Ma, Solid state Commun., 112, 251, (1999). 
12. H. H. Hassan, E. M. Abdel Barry, A. M. El-Lawindy, M. Abo-assy and F. El-Tantawy,Polymer International,30(3),371,(1993)

13. F. El-Tantawy and F. S.Deghaidy, Polymer International, 49,1-6(2000)

14. A.A. Hashem, J. of Applied polymer Sci., 42, 1082, (1991).

15. A. licea Claerei and F. J. U. Carrillo, Polymer Testing, 16(5), 445, (1997).

16. K.M. Sumita, J. Material Sci., 31, 281, (1996).

\section{Appendix}

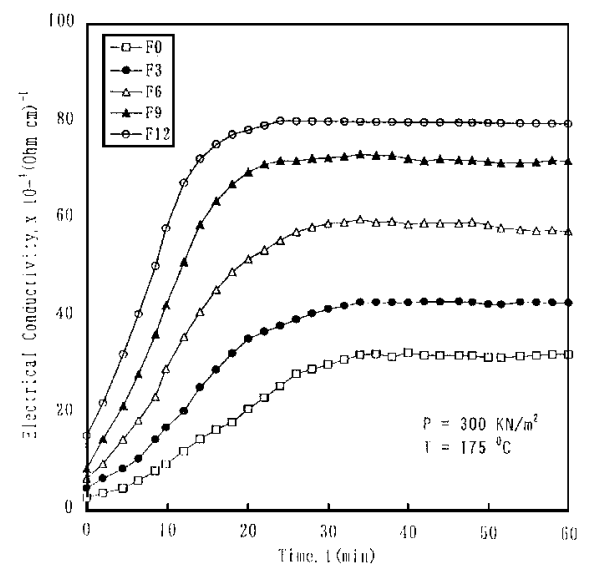

Fig.(1):The vulcanization time versus electrical conductivity of IIR composites with different concentrations of $\mathrm{CF}$ at $\mathrm{P}=300$ $\mathrm{kN} / \mathrm{m} 2$ and $\mathrm{T}=175{ }^{\circ} \mathrm{C}$.

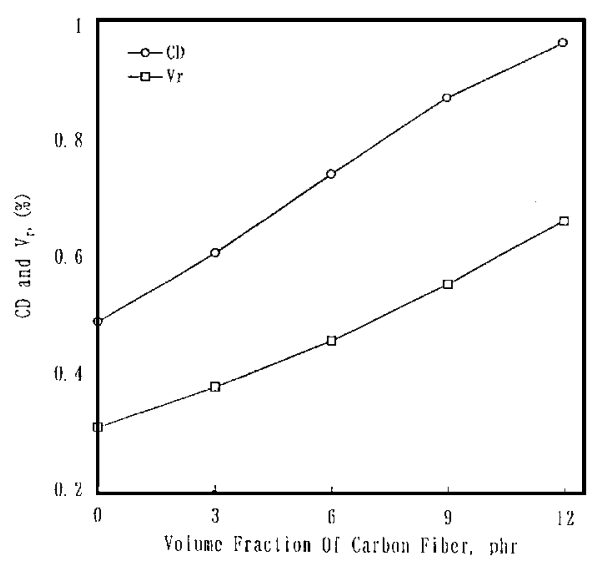

Fig.(2): Shows the relationship between crosslinking density (CD) and volume fraction of rubber ( $\mathrm{Vr}$ ) against $\mathrm{CF}$ content. 


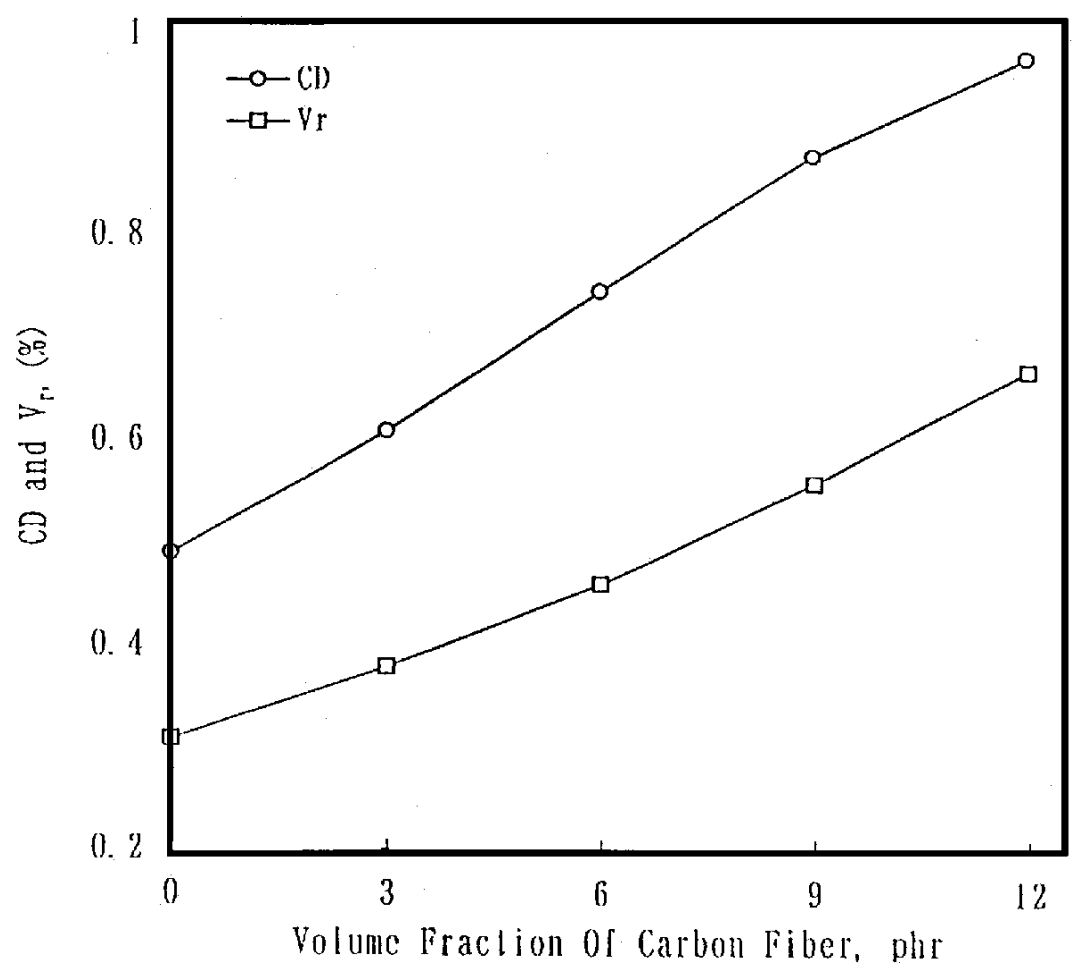

Fig.(3): Shows the effect of $\mathrm{CF}$ on the electric resistance of IIR composites during swelling in kerosene at $20^{\circ} \mathrm{C}$.


Fig.(4): SEM photograph for sample (F0) and sample (F6) after swelling in kerosene for 5 days. 




Fig.(5): Shows the mass swelling of IIR composite as a function of CF constant.

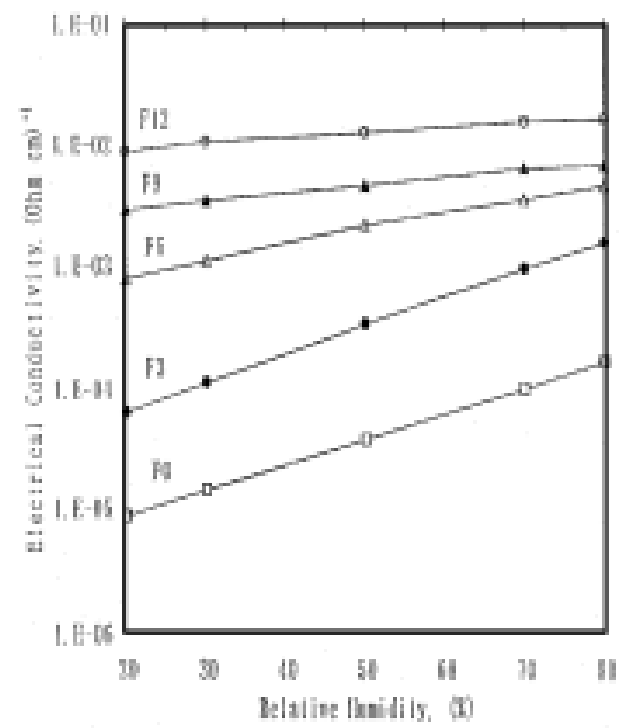

Fig.(6): Shows the relationship between relative humidity of IIR composites and electric resistance as a function of CF content. 

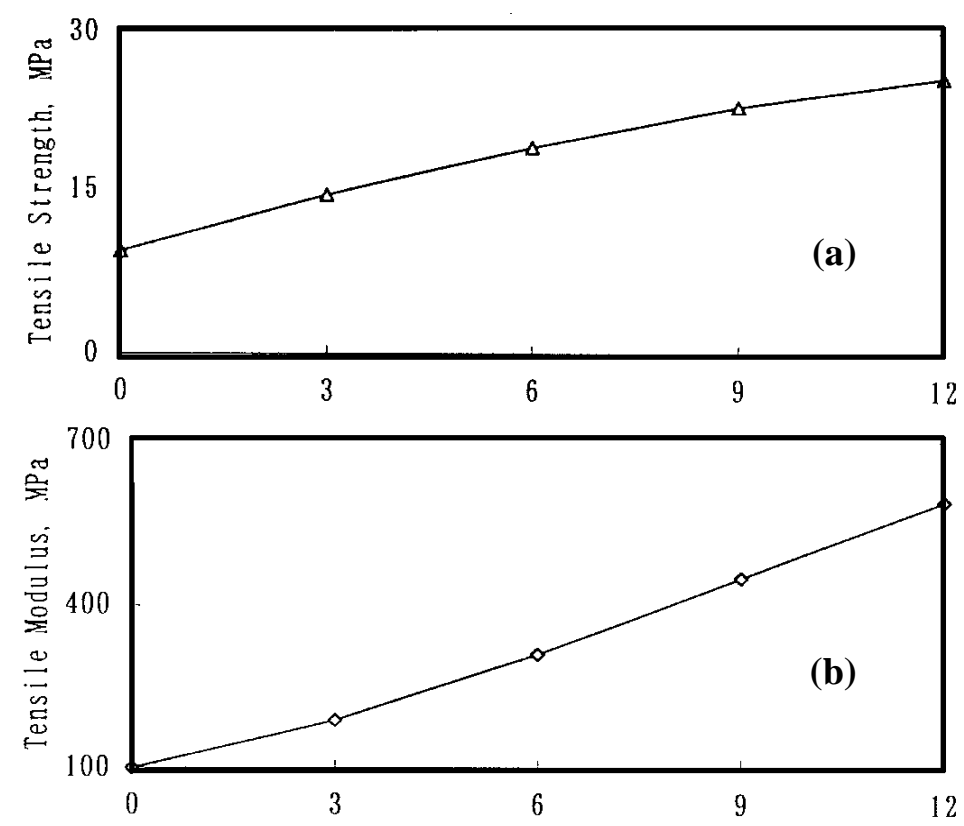

Fig.(7): Tensile strength and tensile modulus of IIR composites as a function of CF content.
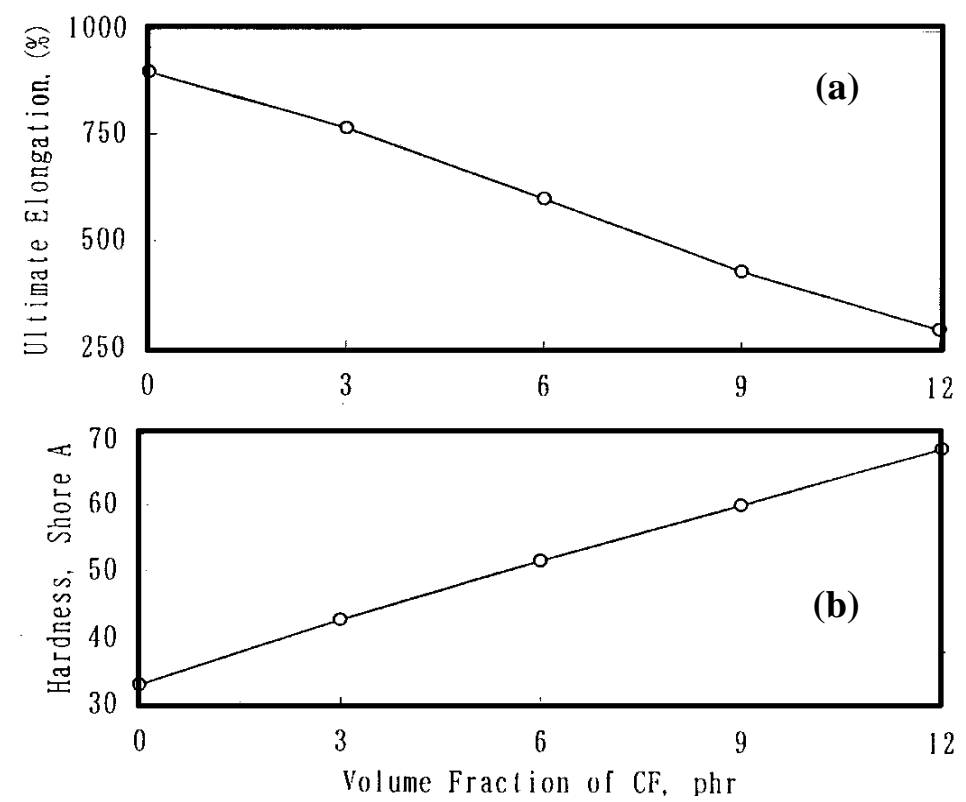

Fig.(8): Optimum elongation and hardness of IIR composites at different $\mathrm{CF}$ content. 